\title{
Transverse Patterns in the Bistable Resonant Tunneling Systems Under Ballistic Lateral Transport
}

\author{
V. A. KOCHELAP ${ }^{\mathrm{a}, \mathrm{b}}$, B. A. GLAVIN ${ }^{\mathrm{a}, \mathrm{b}}$ and V. V. MITIN ${ }^{\mathrm{b}, *}$ \\ ${ }^{a}$ Institute of Semiconductor Physics, Ukrainian Academy of Sciences, Pr. Nauki 45, \\ Kiev 252028, Ukraine; ${ }^{\mathrm{b}}$ Department of Electrical and Computer Engineering, Wayne State University, \\ Detroit, MI 48202, USA
}

\begin{abstract}
We report the theoretical investigation of the phenomenon of the formation of patterns transverse to the tunneling current in resonant tunneling double-barrier heterostructures in the case of wide range of bistable voltages. In contrast to the case of the patterns in the structures with small region of bistability, for pronounced bistability electron lateral transport is strongly nonlocal. We performed numerical simulations of the stationary and mobile patterns using special variational procedure. Our results revealed that though the possible types of patterns remains the same as for the structures with small bistability region, their characteristics are modified considerably.
\end{abstract}

Keywords: Resonant tunneling, bistability, patterns, ballistic transport

\section{INTRODUCTION}

It is well established that the resonant tunneling in the double barrier heterostructures $(\mathrm{DBH})$ is supplemented by the dynamic charge accumulation in the quantum well. This charge accumulation is particularly substantial in the case of the structure with asymmetrical barriers. The important effect, induced by the built-up charge is intrinsic bistability of the system under consideration. For some range of biases two stable states exist at the same bias. One state is characterized by a large built-up charge, resonant tunneling conditions and a large current, the other one corres- ponds to resonance breaking, lowering of the quasi-bound state below the bottom of the emitter band and a low charge built-up and current. The effect of bistability transforms the shape of the current-voltage characteristic of the resonant tunneling diode from $N$-type to $Z$-type. The effect of bistability was experimentally observed in [1-3]. Theoretical investigations of the bistability were performed in [4-6]. In these papers, the tunneling was considered as one-dimensional and the transport through the DBH was supposed to be dependent on only one coordinate, perpendicular to the barriers. Actually, most of the DBH are layered ones and a tunneling electron moves

\footnotetext{
* Corresponding author.
} 
not only across the layers (vertical transport), but also along these layers (horizontal, or lateral, transport). In previous works $[7,8]$ we have shown that under the bistable conditions not only laterally uniform, but also nonuniform configurations of built-up charge and tunneling current (patterns) can exist. Results of [7, 8] were applicable mainly to DBHs with a small range of bistable voltages, where local approach for electron lateral transport was applied. In this work we present results of numerical simulations of stationary and mobile patterns for the DBHs with wide voltage range of bistability, where electron lateral transport is ballistic and strongly nonlocal.

\section{MODEL AND BASIC EQUATIONS}

Since the problem of the transverse patterns requires at least a two dimensional spatial analysis, we use a simple model, showing the main features of the bistability and the patterns. We deal with the model of a resonant tunneling heterostructure, schematically shown in Figure 1. The structure is treated as a system of three parts, weakly coupled by tunneling: emitter $(E)$, quantum well $(Q W)$ and collector $(C)$. The electrodes $E$ and $C$ are usually heavy doped semiconductors and are supposed to

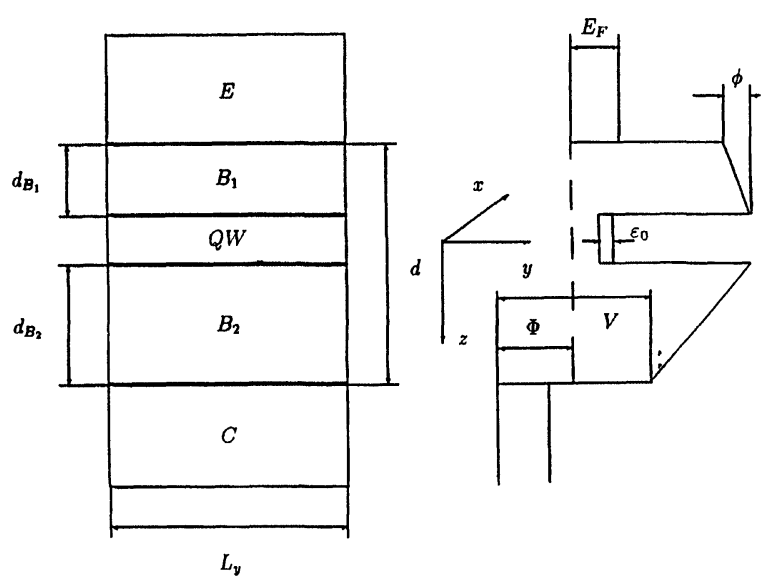

FIGURE 1 The scheme of the resonant tunneling structure and its energy band diagram under bias. be ideal conductors. The energy height of the barriers $B_{1}, B_{2}$ is $V$ and their thicknesses are $d_{B_{1}}, d_{B_{2}}$, respectively. Charge accumulation in the well causes a change of the potential profile in the whole structure. It alters the position of the quasibound state with respect to the bottom of the energy band of the emitter and, in general, with respect to the bottom of the quantum well. We disregard the latter effect and consider, that the built-up charge shifts equally the well bottom and the quasi-bound level. Such a case corresponds to the very thin quantum well, where the built-up charge can be accounted for as an infinitely thin sheet.

The horizontal electron transfer is the main process, determining the transverse patterns. This transfer can be thought as follows. The electron is injected from the emitter to the well in general with a finite horizontal component of the momentum $p$ or velocity $v=p / m^{*}$ ( $m^{*}$ is effective mass). The velocity depends on the position of the quasibound state energy with respect to the Fermi energy $E_{F}$ in the emitter: when the energy quasibound level, $\varepsilon_{0}$, moves from $E_{F}$ through the bottom of the emitter band $E_{0}$, the velocity changes from zero to the Fermi velocity $v_{F}=\sqrt{2 E_{F} / m^{*}}$. For estimates, one can account that $v$ and $v_{F}$ have the same order of magnitude. We can introduce the characteristic time for horizontal transfer: time of tunneling escape from the well $\tau_{\text {es }}$. As we show below, the existence of the developed intrinsic bistability is strongly related with the character of electron lateral transport. Namely, the time of electron tunneling escape from the $Q W$ should be smaller than the time of scattering on phonons, impurities, etc. It means, that between the tunneling events electron moves in the $Q W$ ballistically. As a result, the horizontal characteristic distance is $L_{\mathrm{ch}}=v \tau_{\mathrm{es}}$. One can expect that the scale of the patterns in question is of the order of $L_{\mathrm{ch}}$.

For narrow resonant level from the uncertainty relation we can write $\varepsilon_{0} \tau_{\text {es }} \gg \hbar$. Combining this inequality with the fact, that $E_{F}, \varepsilon_{0}$ and the kinetic energy of the horizontal motion $m^{*} v^{2} / 2$ are of the same order of magnitude, we find for the in-plane 
wave vector $k$ :

$$
k L_{\mathrm{ch}}=\frac{p}{\hbar} L_{\mathrm{ch}}=\frac{m^{*} v^{2}}{\hbar} \tau_{\mathrm{es}} \sim \varepsilon_{0} \tau_{\mathrm{es}} \gg 1 .
$$

The latter estimate shows, that horizontal transfer can be considered as classical, while the vertical transport should be treated as quantum. As a result, we can introduce the distribution function of the resonant electrons in the $Q W$. Assuming weak tunneling coupling between $E, Q W$ and $C$, we derive the kinetic equation for the distribution function $f$ :

$$
\frac{\partial f}{\partial t}+\frac{\vec{p}}{m^{*}} \frac{\partial f}{\partial \vec{r}}-\frac{\partial \phi}{\partial \vec{r}} \frac{\partial f}{\partial \vec{p}}=G(\phi(\vec{r}, t), \vec{p})-\frac{f}{\tau_{\mathrm{es}}}+I\{f\}
$$

where $\phi(\vec{r})$ is the electrostatic potential energy in the well, $G(\phi(\vec{r}, t), \vec{p})$ is the local rate of tunneling from the emitter to the well, $\tau_{\text {es }}$ is the tunneling escape time, $I\{f\}$ is the collision integral for the electrons inside the well, $G$ and $\tau_{\text {es }}$ are functions of $\phi$ at fixed $\vec{r}$. They are expressed through the tunneling probabilities and the Fermi distribution of electrons in the emitter.

From the uncertainty condition (1) we can deduce, that the characteristical scale $L_{\mathrm{ch}}$ greatly exceeds the well width. We assume $L_{\mathrm{ch}}$ is much larger than the thickness of the DBH: $L_{\mathrm{ch}} \gg d$. In this case one can use the quasi-local relation between $\phi$ and electron density $n \equiv \Sigma_{\vec{p}} f(\vec{r}, \vec{p}, t)$ :

$$
\left(\phi(\vec{r})+\frac{d_{B_{1}}}{d} \Phi\right) \frac{\kappa}{4 \pi e^{2}} \frac{d}{d_{B_{1}} d_{B_{2}}}=n(\vec{r}),
$$

which can be derived from the Poisson equation. Here $\kappa$ is dielectric permittivity, $\Phi$ is the external bias in the energetical units. Eqs. (2), (3) compose the system of coupled nonlinear equations which describe the system under consideration.

\section{BISTABILITY IN THE UNIFORM STRUCTURE}

Let us show, that the model formulated above allows the bistable vertical transport regimes with uniform tunneling in the $x, y$ plane. In such a case the $\vec{r}, t$ dependences are absent and from kinetic Eq. (2) one can find the areal electron concentration

$$
n(\phi)=n_{0}(\phi) \equiv \tau_{\mathrm{es}}(\phi) \sum_{\vec{p}} G(\phi(\vec{r}, t), \vec{p}) .
$$

Since the left-hand side of (4) is a function of $\phi$, we get two algebraic Eqs. (3) and (4) for two variables $n, \phi$. It is convenient to rewrite this system as

$$
L(\phi) \equiv n_{0}(\phi)=\frac{\kappa d}{4 \pi e^{2} d_{B_{1}} d_{B_{2}}}\left(\phi+\frac{d_{B_{1}}}{d} \Phi\right) \equiv R(\phi) .
$$

For the particular heterostructure the latter equation has one controlling parameter, external bias $\Phi$.

We have calculated the functions $\tau_{\text {es }}(\phi)$ and $g(\phi)$ for the heterostructure with parameters: $V=1 \mathrm{eV}$, $m^{*}=0.067 m_{0}, d=5.7 \mathrm{~nm}, d_{B_{1}}=2 \mathrm{~nm}, \varepsilon_{0}=0.1 \mathrm{eV}$, $\kappa=11.5$. In Figure 2 the left and right-hand sides are shown for $E_{F}=56 \mathrm{meV}$ and zero temperature. The dependence of $L$ on $\Phi$ is weak and only one curve $L(\phi)$ is shown. Qualitatively the dependence $L(\phi)$ corresponds to the switching on of the resonant tunneling when the resonant level crosses the bottom of emitter conduction band and further gradual decrease of the number of resonant electrons in the emitter while the resonant level is shifted toward the Fermi level of the emitter. $R(\phi)$ is just a straight line whose vertical shift is determined by the bias. From Figure 2 one can see that our system possess the property of bistability in the particular range of biases $\Phi_{l}<\Phi<\Phi_{h}$. Corresponding current-voltage characteristic is shown in the insert of Figure 2.

The approach, used in Eq. (2) allows to include into the tunneling generation rate $G$ the effect of scattering of the resonant electrons in the $Q W$ (see [9]). The analysis of this effect has shown that the wide range of the bistability can take place if $\tau_{\text {ew }} \ll \tau_{\text {sc }} \tau_{\text {cw }} /\left(\tau_{\text {cw }} \tau_{\text {sc }}\right)$. Here $\tau_{\text {ew }}, \tau_{\text {cw }}$ are the char- 


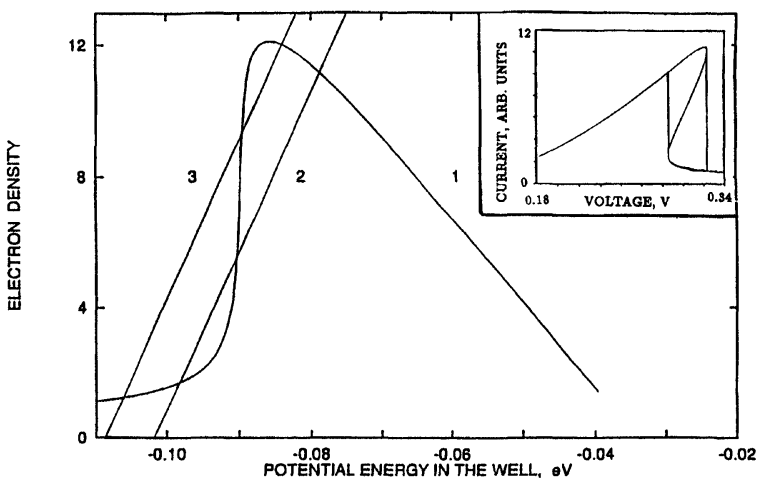

FIGURE 2 Illustration of the self-consistent solutions of the bistability problem under uniform tunneling for the structure described in the text. Curve 1 is almost independent on the external bias $L(\phi)$ and other curves are $R(\phi)$ for two biases: $0.29 \mathrm{eV}$ (curve 2) and $0.31 \mathrm{eV}$ (curve 3 ). The insert shows the current-voltage characteristic. $L$ and $R$ are in units $10^{11} \mathrm{~cm}^{-2}$.

acteristical times of tunneling from the emitter to the $Q W$ and from the $Q W$ to the collector, calculated for the electrons with the energy of vertical motion equal to the Fermi energy in the emitter, and $\tau_{\mathrm{sc}}$ is the characteristical time of scattering. It means, that the bistable range of voltages is substantial for the structure with high asymmetry in the barriers transmission coefficients (the collector barrier should be less transparent) and with ballistic or quasi-ballistic character of lateral electron transport. Furthermore, this fact explains dependence of the bistability on the temperature, which is usually observed in experiments: at higher temperatures $\tau_{\text {sc }}$ decreases and this washes out the asymmetry of tunneling in the system and the effect of bistability.

\section{PATTERNS UNDER BALLISTIC REGIME OF HORIZONTAL TRANSFER}

We consider the theory of the one-dimensional patterns, in which all physical values (built-up charge, tunneling currents, etc.) depend on only one coordinate, namely $y$. Then, we assume completely ballistic lateral electron transport. In this case (2) becomes

$$
\frac{\partial f}{\partial t}+\frac{p}{m^{*}} \frac{\partial f}{\partial y}-\frac{\partial \phi}{\partial y} \frac{\partial f}{\partial p}=G-\frac{f}{\tau_{\mathrm{es}}} .
$$

here $p$ labels the $y$-component of momentum $\vec{p}$. One can solve (6) in terms of the characteristic curves

$$
p= \pm \sqrt{p_{0}^{2}+2 m^{*}\left(\phi\left(y_{0}\right)-\phi(y)\right)} \equiv \mathcal{P}\left(p_{0}, y_{0}, y\right)
$$

where $p_{0}$ is the momentum of the electron, injected into the well at the point $y=y_{0}$. The general solution of the kinetic equation has the form

$$
\begin{gathered}
f(y, \vec{p})=\int m^{*} \frac{d y^{\prime}}{\mathcal{P}\left(p, y, y^{\prime}\right)} M\left(p, y, y^{\prime}\right) \\
G\left(\mathcal{P}\left(p, y, y^{\prime}\right), y^{\prime}\right)
\end{gathered}
$$

where the kernel $M\left(p, y, y^{\prime}\right)$ depends on the particular shape of the potential $\phi(y)$ and can be easily calculated in the explicit form.

Unfortunately, iteration methods of solution fail in the solution of (3), (8) due to stability problems. Because of that we have applied the following variational procedure for the self-consistent solution of (3), (8). We introduce the functional

$$
J\{\phi\}=\int d y(\phi-\mathcal{L}\{\phi\})^{2},
$$

where

$$
\mathcal{L}\{\phi\}=-\frac{d_{B_{1}}}{d} \Phi+\frac{4 \pi e^{2} d_{B_{1}} d_{B_{2}}}{\kappa d} m^{*} \sum_{\vec{p}} \int \frac{d y^{\prime}}{\mathcal{P}} M G .
$$

Functional $J$ equals zero for the exact solution of (3), (8). For a particular solution we can choose some probe functions $\phi_{\mathrm{pr}}\left(y, c_{i}\right)$, where $c_{i}$ are variational parameters. These parameters are determined by the condition of minimization of $J\left(c_{i}\right)$. It can be shown that our variational formulation of the problem of patterns is equivalent to the initial system of Eqs. (3), (6). 
Using this method we have analyzed the possible types of stationary patterns. The possible types of patterns are the same as for the case of the structures with small range of bistable biases. The bistable range of biases can be split into the two regions $\Phi_{l}<\Phi<\Phi_{c}$ and $\Phi_{c}<\Phi<\Phi_{h}$ with the different types of patterns. In the first region the soliton-like patterns can exist. In these patterns at large $|y|$ the system is in the low-current state, while in the certain spatial region the local increase of the built-up charge and tunneling current takes place. At $\Phi_{c}<\Phi<\Phi_{h}$ the possible patterns are anti-soliton-like with the opposite characteristics: at high $|y|$ the system is in the high current state and local decrease of the built-up charge and the tunneling current in the finite spatial region. The $\Phi=\Phi_{c}$ is the critical bias: in this case the special kink-like pattern occurs. In this pattern high and low current states coexist in the different spatial regions.

Our calculations revealed that the anti-soliton patterns are substantially wider than soliton patterns. This is because for soliton pattern in the region of nonuniformity tunneling via both barriers is possible, while for anti-soliton only tunneling via collector barrier takes place. This influence the characteristical length which an electron can pass in the quantum well, and consequently the width of the pattern. The degree of the asymmetry in the spatial scales of the soliton anti-soliton patterns strongly depends on the degree of asymmetry of the transmission coefficients of emitter and collector barriers. To avoid numerical difficulties, we performed numerical simulations of the stationary patterns for a structure with thinner collector barrier with respect to the structure described in Section 3, namely with $d_{B_{2}}=3.4 \mathrm{~nm}$. The dependence of the $L_{s}$ for the case of solitonlike pattern and $L_{a}$ for the case of anti-soliton pattern on the dimensionless parameter $q \equiv\left(\Phi-\Phi_{l}\right) /\left(\Phi_{h}-\Phi_{l}\right)$ is presented in Figure 3 .

The important property of patterns in the case of pronounced bistability is substantial difference between the spatial regions of localization of nonuniformities of the emitter- $Q W$ and the $Q W$ -

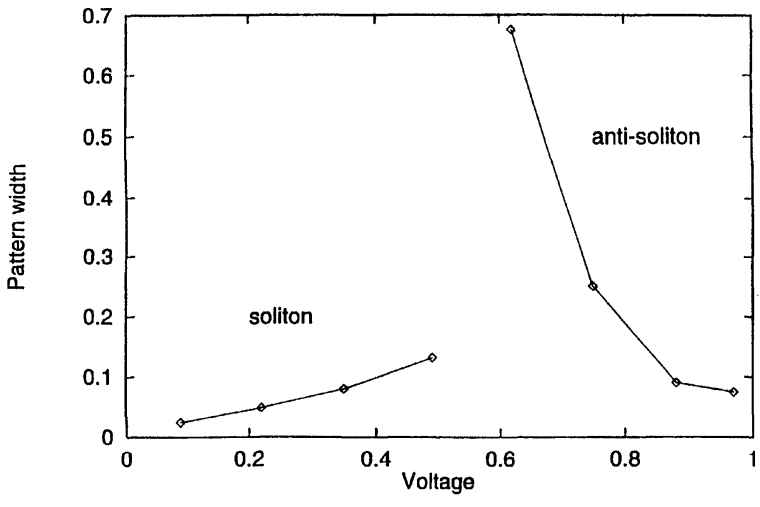

FIGURE 3 Dependence of the width of the soliton pattern, $L_{s}$, and anti-soliton pattern, $L_{a}$, on the dimensionless voltage parameter $q \equiv\left(\Phi-\Phi_{l}\right) /\left(\Phi_{h}-\Phi_{l}\right)$.

collector tunneling currents. This is due to i) a strong dependence of the tunneling injection rate from the emitter to the $Q W$ on the position of the resonant level with respect to the bottom of the emitter conduction band and ii) a ballistic leakage of the injected electrons over the $Q W$ before the tunneling escape to the contacts. This is illustrated in Figure 4. In the upper part of the figure the current field in the structure is shown (spacing between the current lines is proportional to the value of the current density). In the lower part of the figure spatial dependences of the emitter- $Q W$,

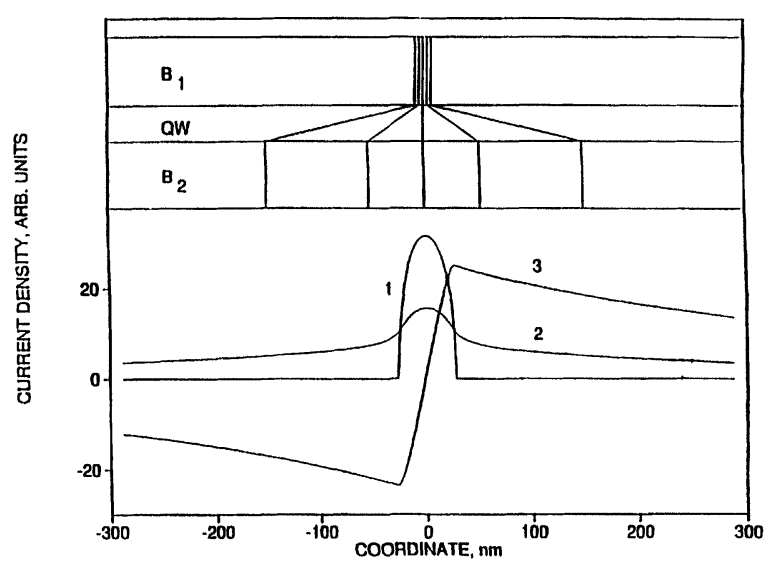

FIGURE 4 The spatial dependence of the current for the soliton-like pattern at $q=0.3$. In the upper part the current field is depicted. In the lower part the emitter (curve 1), collector (curve 2, multiplied by factor 5) and two-dimensional lateral (curve 3) currents are presented. 
$Q W$-collector and two-dimensional lateral currents are depicted. The bias at which calculations were performed corresponds to $q=0.3$.

In addition to the stationary, we have investigated mobile autowave patterns. In fact, they are the moving kink-like patterns, describing switching of the DBH from one uniform state to the other. For the particular value of the bias the unique value of the switching wave velocity and the type of the switching exist. Namely, for $\Phi_{l}<\Phi<\Phi_{c}$ the switching from the high to the low current state is possible, while for $\Phi_{c}<\Phi<\Phi_{h}$ the switching from the high to the low current state can occur. Due to the ballistic character of the horizontal transport the characteristical velocity of the switching waves is determined by the Fermi velocity of electrons in the emitter $v_{F}$. In Figure 5 the dependence of the switching wave velocity (in units $v_{F}$ ) on $q$ is presented for the structure described in Section 3. The insert shows the spatial dependence of the built-up charge in the stationary kink at $\Phi=\Phi_{c}$. The positive sign of velocity, in accordance with mentioned above, corresponds to the switching from the high to the low current state.

Note, that (3) is obtained for the infinitely conducting emitter and collector. For autowaves, moving with the velocity of the order of $v_{F}$ and

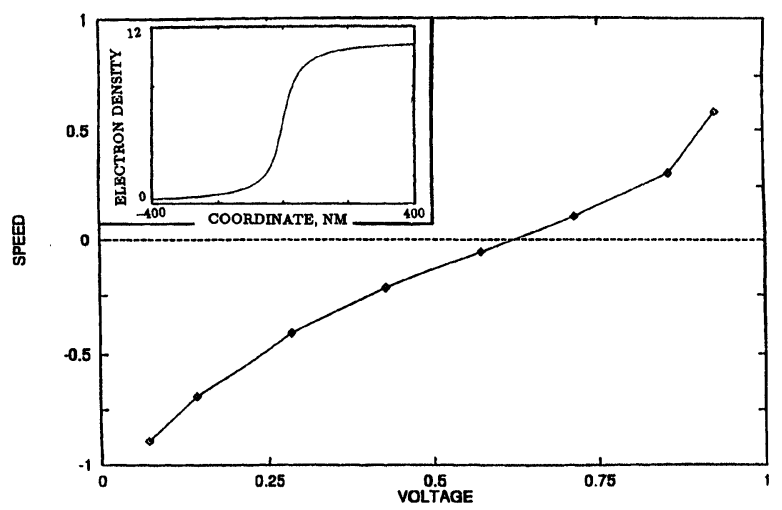

FIGURE 5 The dependences of the switching wave velocity (in units $v_{F}$ ) on dimensionless voltage parameter $\left(\Phi-\Phi_{l}\right) /$ $\left(\Phi_{h}-\Phi_{l}\right)$. The spatial dependence of the built-up charge (in units $10^{11} \mathrm{~cm}^{-2}$ ) in the stationary kink is shown in the insert. spatial scale of the kink transition region $v_{F} \tau_{\text {es }}$, this assumption is valid for the structures, in which $\tau_{\text {es }}$ is substantially greater than the characteristical time of relaxation in electrodes. This is true for the heterostructures with thick enough barriers and electrodes with high conductivity. Otherwise, the characteristics of autowaves (their velocity, for example) can be modified by the relaxation processes in the electrodes.

\section{SUMMARY}

In a conclusion, we have investigated the phenomenon of the transverse patterns formation in the resonant tunneling double barrier diode with the wide voltage range of intrinsic bistability of the current-voltage characteristic. These patterns are stationary or mobile nonuniform distributions of the built-up charge and tunneling current. For the pronounced bistability the characteristics of the patterns are determined by the ballistic and nonlocal character of lateral transport of the resonant electrons in the $Q W$. This fact gives rise to specific features of the patterns with respect to the similar phenomena in DBHs with a small range of bistable voltages. For numerical simulations a special variational procedure was developed.

\section{Acknowledgements}

The authors would like to thank Drs. J. Schulman, F. Vasko and V. Sheka for discussions.

This work was supported by US ARO and by the Ukrainian State Committee for Science and Technology (grant No. 2.2/49).

\section{References}

[1] Goldman, V. J., Tsui, D. C. and Cunningham, J. E. (1987). "Observation of Intrinsic Bistability in Resonant Tunneling Structures", Phys. Rev. Lett., 58, 1256.

[2] Leadbeater, M. L., Alves, E. S., Eaves, L., Henini, M., Hughes, O. H., Sheard, F. W. and Toombs, G. A. (1988). 
"Charge Build-up and Intrinsic Bistability in Resonant Tunneling Structures", Semicond. Sci. Technol., 3, 1060.

[3] Zaslavski, A., Goldman, V. J., Tsui, D. C. and Gunningham, J. E. (1988). "Resonant Tunneling and Intrinsic Bistability in Asymmetric Double Barrier Heterostructures", Appl. Phys. Lett., 53, 1408.

[4] Sheard, F. W. and Toombs, G. A. (1988). "Space Charge Buildup and Bistability in Resonant Tunneling Double Barrier Structures", Appl. Phys. Lett., 52, 1228.

[5] Kluksdhal, N. C., Kriman, A. M., Ferry, D. K. and Ringhofer, C. (1989). "Selfconsistent Study of the Resonant Tunneling Diode", Phys. Rev. B, 39, 7720.

[6] Jensen, K. and Buot, F. (1991). "Numerical Simulations of Intrinsic Bistability and High Frequency Current Oscillations in Resonant Tunneling Structures", Phys. Rev. Lett., 66, 1078.

[7] Kochelap, V. A., Glavin, B. A. and Mitin, V. V. (1996). "Patterns in Bistable resonant Tunneling Systems", in Hot Carriers in Semiconductors, ed. by K. Hess, J.-P. Leburton and U. Ravaioli, New York: Plenum Press, p. 551.

[8] Glavin, B. A., Kochelap, V. A. and Mitin, V. V. (1996). "Patterns in Bistable resonant Tunneling Diode: Possibility of Novel Electron Device", Proc. of the International Conference on Quantum Device and Circuits, Imperial College Press, p. 170.

[9] Iannaccone, G. and Pellegrini, B. (1995). "Density of States in Double Barrier Resonant Tunneling Systems", Phys. Rev. B, 53, 2020.

\section{Authors' Biographies}

V. A. Kochelap Viacheslav Kochelap received M.S. degree from Kiev State University (Ukraine) and $\mathrm{Ph} . \mathrm{D}$. degree from Institute of Semiconductors (Kiev, Ukraine). At present he is chairman of the
Department of Theoretical Physics of the Institute of Semiconductors (Kiev, Ukraine).

His research interests are in the field of theory of semiconductors and semiconductor devices, electron-phonon interaction, electron transport and noise in low-dimensional structures, quantum electronics, nonlinear optics.

B. A. Glavin Boris Glavin received M.S. degree from Kiev State University. Currently he is working on his Ph.D. dissertation in Electrical Engineering. He works in the field of nonlinear effects in resonant tunneling semiconductor heterostructures and theory of phonons in low-dimensional semiconductor structures.

V. V. Mitin Vladimir Mitin received M.S. degree from Rostov State University (Russia) and Ph.D. degree from Institute of Semiconductors (Kiev, Ukraine). Currently he is professor of Electrical and Computer Engineering at Wayne State University, Detroit, USA.

His scientific activities are in the sphere of electronic and optoelectronic devices and materials, simulations of electron transport and noise in low dimensional semiconductor structures, growth of semiconductors, heat removal in semiconductor devices. 

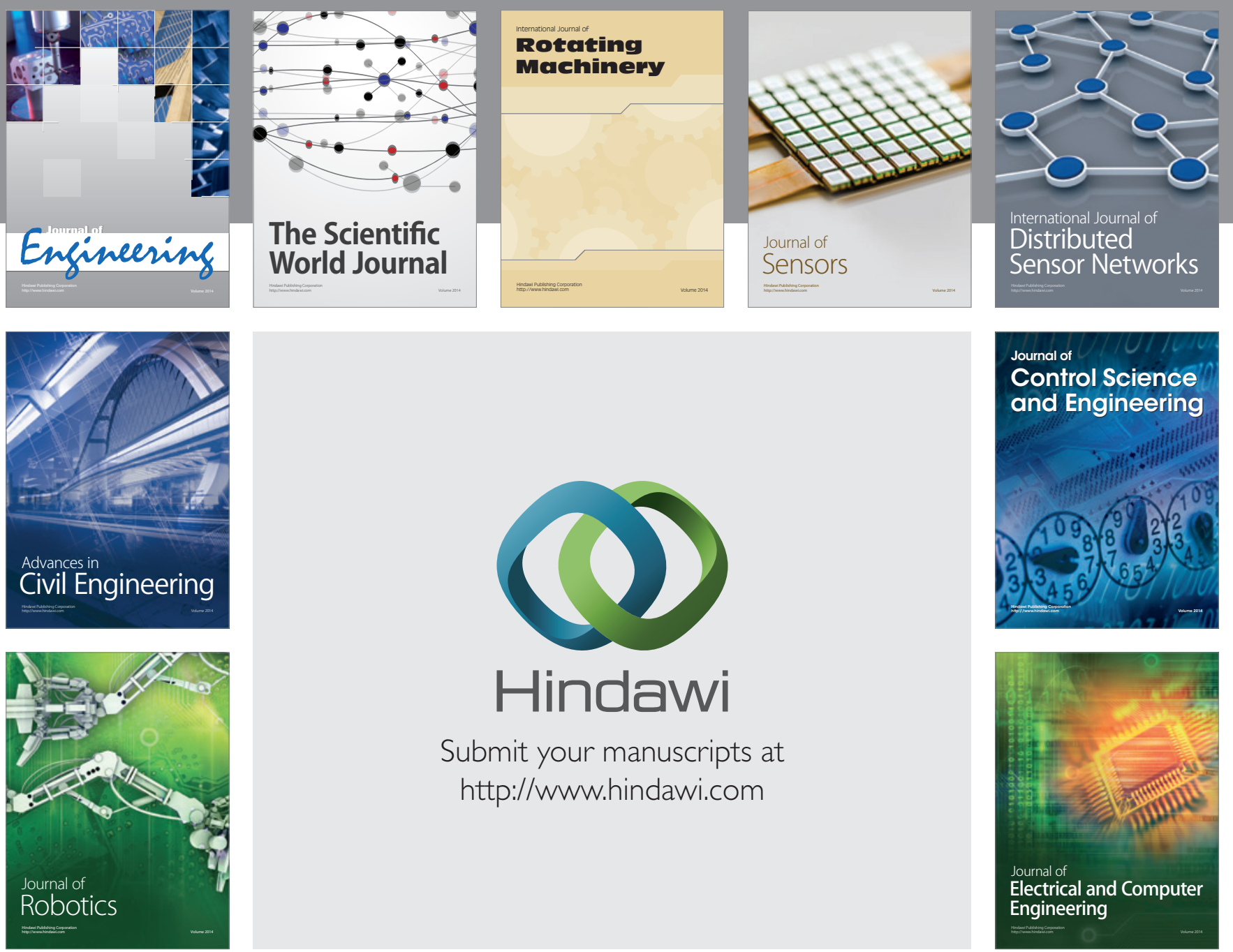

Submit your manuscripts at

http://www.hindawi.com
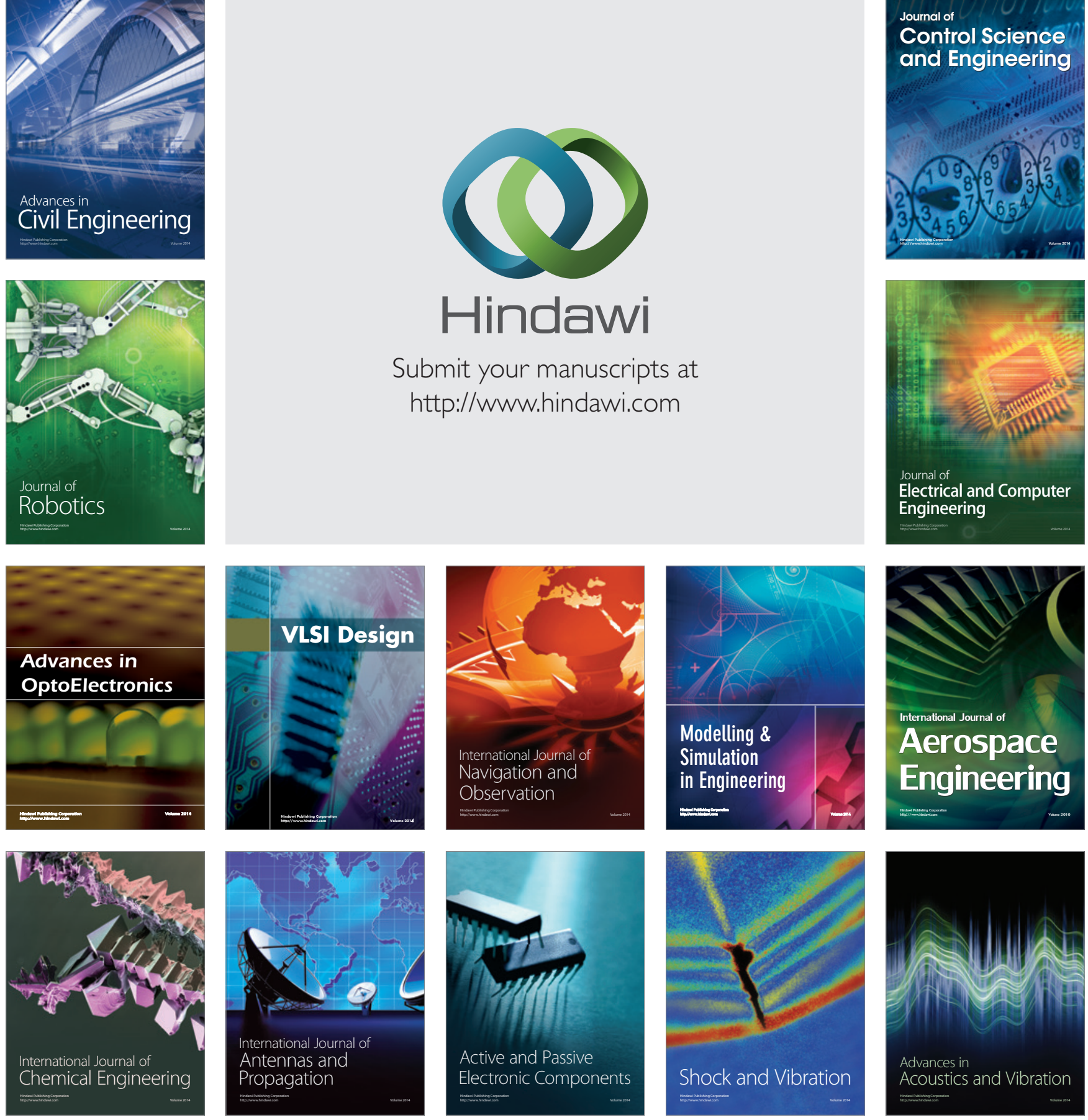